\title{
FAMOUS ARTISTS WHO SUFFER(ED) FROM RHEUMATIC DISEASES: A SYSTEMATIC REVIEW
}

Jozélio Freire de Carvalho ${ }^{1, \star}$, Felipe Freire da Silva², Carlos Augusto Ferreira de Andrade ${ }^{3,4}$, José Dirson Argolo ${ }^{5,6}$, Licia Maria Henrique da Mota7

1.Universidade Federal da Bahia, Salvador (BA), Brazil. 2.Universidade de São Paulo, São Paulo (SP), Brazil. 3.Fundação Oswaldo Cruz, Rio de Janeiro (RJ), Brazil. 4.Universidade de Vassouras, Rio de Janeiro (RJ), Brazil. 5.Universidade Federal da Bahia, Salvador (BA), Brazil. 6.Stúdio Argolo, Salvador (BA), Brazil.7.Universidade de Brasília, Brasília (DF), Brazil.

*Corresponding author: jotafc@gmail.com

\section{BACKGROUND}

This paper aims to review articles that discuss famous artists who have/had suspected or confirmed diagnosis of rheumatic diseases.

\section{MATERIALS AND METHODS}

We have systematically researched articles published in PubMed, MEDLINE, LILACS and SciELO dating from 1965 to June 2020. Our search strategy employed the keywords: "art", "artist”, "rheumatology", "rheumatism", "rheumatic diseases" and "arthritis"; "arthritis", "rheumatoid arthritis", "spondyloarthritis", "gout", "systemic sclerosis" and "systemic lupus erythematosus". The authors did not limit the search to articles published in any language nor of any study design.

\section{RESULTS}

We identified 19 famous artists who suffered rheumatic diseases. The majority of them had rheumatoid arthritis $(n=8)$, gout $(n=4)$, systemic sclerosis $(n=2)$, reactive arthritis $(n=2)$, juvenile rheumatoid arthritis $(n=1)$, ankylosing spondylitis $(n=1)$, fibromyalgia $(n=1)$, pycnodysostosis $(n=1)$ and pustulotic arthro-osteitis syndrome $(n=1)$. Confirmed diagnosis was verified in $8 / 19$ and presumptive diagnosis in 11/19. All of them continued to perform their artistic activities despite the limitations secondary to rheumatic diseases. Importantly, the most (14/19) of these artists had an art style changing after the beginning of the rheumatic condition. Treatments varied from nonpharmacological therapies such as diets $(n=3)$, spas $(n=4)$, exercises/physical therapies $(n=4)$, surgeries $(n=2)$, occupational therapy $(n=2)$, synoviorthesis with yttrium $(n=1)$ and use of drugs such as antipyretics and anti-inflammatory drugs $(n=6)$, cortisone $(n=2)$, methotrexate $(n=1)$, antimalarials $(n=2)$, gold $(n=2)$, colchicine $(n=1)$, homeopathy $(n=2)$ until biologicals $(n=1)$.

\section{CONCLUSION}

There are several reports regarding famous artists who have or had rheumatic diseases described in the scientific literature. Herein, the authors review all these interesting described cases. 